\title{
Spin polarized neutron matter and magnetic susceptibility within the Brueckner-Hartree-Fock approximation
}

\author{
I. Vidaña, ${ }^{1,2}$ A. Polls, ${ }^{2}$ and A. Ramos ${ }^{2}$ \\ ${ }^{1}$ Dipartimento di Fisica, Università di Pisa and INFN Sezione di Pisa, Via Buonarroti 2, I-56127 Pisa, Italy \\ ${ }^{2}$ Departament d'Estructura i Constituents de la Matèria, Universitat de Barcelona, E-08028 Barcelona, Spain
}

(Received 20 November 2001; published 22 February 2002)

\begin{abstract}
The Brueckner-Hartree-Fock formalism is applied to study spin polarized neutron matter properties. Results of the total energy per particle as a function of the spin polarization and density are presented for two modern realistic nucleon-nucleon interactions, Nijmegen II and Reid93. We find that the dependence of the energy on the spin polarization is practically parabolic in the full range of polarizations. The magnetic susceptibility of the system is computed. Our results show no indication of a ferromagnetic transition which becomes even more difficult as the density increases.
\end{abstract}

DOI: 10.1103/PhysRevC.65.035804

PACS number(s): 26.60.+c, 21.60.Jz, 26.50.+x

\section{INTRODUCTION}

The study of the magnetic properties of dense matter is of considerable interest in connection with the physics of pulsars. These objects, since the suggestion of Gold [1], are generally believed to be rapidly rotating neutron stars with strong surface magnetic fields of the order of $10^{12}$ Gauss. Several authors have studied the possible existence of a phase transition to a ferromagnetic state on pure neutron systems at densities corresponding to the theoretically stable neutron stars. Brownell and Callaway [2] and Rice [3] considered a hard sphere gas model and showed that the ground state of the neutron gas becomes ferromagnetic at $k_{F}$ $\approx 2.3 \mathrm{fm}^{-1}$. Silverstein [4] and Østgaard [5] found that the inclusion of long range attraction significantly increased the ferromagnetic transition density (e.g., Østgaard predicted the transition to occur at $k_{F} \approx 4.1 \mathrm{fm}^{-1}$ using a simple central potential with hard core only for singlet spin states). Clark [6] and Pearson and Saunier [7] calculated the magnetic susceptibility for low densities $\left(k_{F} \leqslant 2 \mathrm{fm}^{-1}\right)$ using more realistic interactions. Pandharipande et al. [8], using the Reid soft-core potential, performed a variational calculation arriving to the conclusion that such a transition was not to be expected for $k_{F} \leqslant 5 \mathrm{fm}^{-1}$. Early calculations of the magnetic susceptibility within the Brueckner theory were performed by Bäckmann and Källman [9] employing the Reid soft-core potential, and results from a correlated basis function calculation were obtained by Jackson et al. [10] with the Reid $v_{6}$ interaction. A different point of view was followed by Vidaurre et al. [11], who employed neutron-neutron effective interactions of Skyrme type, finding the ferromagnetic transition at $k_{F} \approx 1.73-1.97 \mathrm{fm}^{-1}$.

In connection with the problem of the neutrino diffusion in dense matter, Fantoni et al. [12] have recently employed a new quantum simulation technique [the so-called auxiliary field diffusion Monte Carlo method (AFDMC)] using realistic interactions (based upon the Argonne $v_{18}$ two-body potential [13] plus Urbana IX three-body potential [14]), and have found that the magnetic susceptibility of neutron matter shows a strong reduction of about a factor of 3 with respect its Fermi gas value. They pointed out that such a reduction may have strong effects on the mean free path of a neutrino in dense matter and, therefore, it should be taken into account in the studies of supernovae and proto-neutron stars.

In this work we employ the Brueckner-Hartree-Fock (BHF) approximation, using the realistic Nijmegen II and Reid93 [15] nucleon-nucleon interactions, to study spin polarized neutron matter properties such as the total energy per particle and the magnetic susceptibility. We employ the socalled continuous prescription when solving the BetheGoldstone equation. As shown by Song et al. [16], the effects from three-body clusters are dimished in this prescription. We also explore in this work the dependence of the total energy per particle on the spin polarization, finding that up to a very good approximation this dependence is parabolic.

The paper is organized in the following way. In Sec. II the theoretical background of our calculation is briefly reviewed. The construction of the neutron-neutron $G$ matrices and the calculation of the total energy per particle are shown in Sec. II A, whereas the magnetic susceptibility is determined in Sec. II B. Our results are presented in Sec. III. Finally, a short summary and the main conclusions are given in Sec. IV.

\section{THEORETICAL BACKGROUND}

In this section we briefly show how to evaluate, in the BHF approximation, the total energy per particle and the magnetic susceptibility of a system of neutrons in which we assume that the density of particles with spin up, $\rho^{\uparrow}$, is different from that with spin down, $\rho^{\downarrow}$.

\section{A. Energy per particle}

Our calculation of the total energy per particle starts with the construction of $G$ matrices, which describe in an effective way the interaction between two neutrons, for each one of the spin combinations $(\uparrow \uparrow, \uparrow \downarrow, \downarrow \uparrow$ or $\downarrow \downarrow)$, in the presence of a surrounding medium. They can be obtained by solving the following integral Bethe-Goldstone equations:

$$
G_{\uparrow \uparrow, \uparrow \uparrow}=V_{\uparrow \uparrow, \uparrow \uparrow}+V_{\uparrow \uparrow, \uparrow \uparrow} \frac{Q_{\uparrow \uparrow, \uparrow \uparrow}}{\omega-\epsilon_{\uparrow}-\epsilon_{\uparrow}+i \eta} G_{\uparrow \uparrow, \uparrow \uparrow},
$$




$$
\begin{aligned}
& G_{\downarrow \downarrow, \downarrow \downarrow}=V_{\downarrow \downarrow, \downarrow \downarrow}+V_{\downarrow \downarrow, \downarrow \downarrow} \frac{Q_{\downarrow \downarrow, \downarrow \downarrow}}{\omega-\epsilon_{\downarrow}-\epsilon_{\downarrow}+i \eta} G_{\downarrow \downarrow, \downarrow \downarrow}, \\
& \left(\begin{array}{ll}
G_{\uparrow \downarrow, \uparrow \downarrow} & G_{\uparrow \downarrow, \downarrow \uparrow} \\
G_{\downarrow \uparrow, \uparrow \downarrow} & G_{\downarrow \uparrow, \downarrow \uparrow}
\end{array}\right) \\
& =\left(\begin{array}{cc}
V_{\uparrow \downarrow, \uparrow \downarrow} & V_{\uparrow \downarrow, \downarrow \uparrow} \\
V_{\downarrow \uparrow, \uparrow \downarrow} & V_{\downarrow \uparrow, \downarrow \uparrow}
\end{array}\right)+\left(\begin{array}{cc}
V_{\uparrow \downarrow, \uparrow \downarrow} & V_{\uparrow \downarrow, \downarrow \uparrow} \\
V_{\downarrow \uparrow, \uparrow \downarrow} & V_{\downarrow \uparrow, \downarrow \uparrow}
\end{array}\right) \\
& \times\left(\begin{array}{cc}
\frac{Q_{\uparrow \downarrow, \uparrow \downarrow}}{\omega-\epsilon_{\uparrow}-\epsilon_{\downarrow}+i \eta} & 0 \\
0 & \frac{Q_{\downarrow \uparrow, \downarrow \uparrow}}{\omega-\epsilon_{\downarrow}-\epsilon_{\uparrow}+i \eta}
\end{array}\right) \\
& \times\left(\begin{array}{ll}
G_{\uparrow \downarrow, \uparrow \downarrow} & G_{\uparrow \downarrow, \downarrow \uparrow} \\
G_{\downarrow \uparrow, \uparrow \downarrow} & G_{\downarrow \uparrow, \downarrow \uparrow}
\end{array}\right)
\end{aligned}
$$

In the above expressions the first (last) two subindices indicate the spin projections of the two neutrons in the initial (final) state, $V$ is the bare nucleon-nucleon interaction, $Q$ is the Pauli operator which allows only intermediate states compatible with the Pauli principle, and $\omega$ is the starting energy defined as the sum of single-particle energies, $\epsilon_{\uparrow(\downarrow)}$, of the interacting neutrons. Note that $G_{\uparrow \downarrow, \uparrow \downarrow}$ and $G_{\downarrow \uparrow, \downarrow \uparrow}$ are obtained from a coupled channel equation due to the mixing induced by the interaction. One can equivalently solve the Bethe-Goldstone equation in the spin-coupled basis, where the interaction is diagonal, although in that case the Pauli operator is nondiagonal. However, the conventional angle average of the Pauli operator makes it diagonal, thus reducing the problem to an uncoupled one in each total spin channel.

The single-particle energy of a neutron with momentum $k$ and spin projection $\sigma=\uparrow(\downarrow)$ is given by

$$
\epsilon_{\sigma}=\frac{\hbar^{2} k^{2}}{2 m}+U_{\sigma}(k),
$$

where the single-particle potential $U_{\sigma}(k)$ represents the average field "felt" by the neutron due to its interaction with the other neutrons of the system. In the BHF approximation it is given by

$$
\begin{aligned}
U_{\sigma}(k)= & \operatorname{Re} \sum_{\sigma^{\prime}=\uparrow, \downarrow} \sum_{k^{\prime} \leqslant k_{F}^{\sigma^{\prime}}} \\
& \times\left\langle\vec{k} \vec{k}^{\prime}\left|G_{\sigma \sigma^{\prime}, \sigma \sigma^{\prime}}\left(\omega=\epsilon_{\sigma}+\epsilon_{\sigma^{\prime}}\right)\right| \vec{k} \vec{k}^{\prime}\right\rangle_{\mathcal{A}},
\end{aligned}
$$

where a sum over the two Fermi seas of spin up and down, characterized by $k_{F}^{\uparrow}=\left(6 \pi^{2} \rho^{\uparrow}\right)^{1 / 3}$ and $k_{F}^{\downarrow}=\left(6 \pi^{2} \rho^{\downarrow}\right)^{1 / 3}$, respectively, is performed and the matrix elements are properly antisymmetrized.

Once a self-consistent solution of Eqs. (1) and (3) is obtained, the total energy per particle is easily calculated:

$$
\frac{E}{N}=\sum_{\sigma=\uparrow, \downarrow} \sum_{k \leqslant k_{F}^{\sigma}}\left(\frac{\hbar^{2} k^{2}}{2 m}+\frac{1}{2} U_{\sigma}(k)\right) .
$$

This quantity is a function of $\rho^{\uparrow}$ and $\rho^{\downarrow}$ or, equivalently, of the total density $\rho=\rho^{\uparrow}+\rho^{\downarrow}$ and the spin polarization $\Delta$, defined as

$$
\Delta=\frac{\rho^{\uparrow}-\rho^{\downarrow}}{\rho} .
$$

Note that the value $\Delta=0$ corresponds to nonpolarized or paramagnetic $\left(\rho^{\uparrow}=\rho^{\downarrow}\right)$ neutron matter, whereas $\Delta= \pm 1$ means that the system is totally polarized, i.e., all the spins are aligned in the same direction.

\section{B. Magnetic susceptibility}

The magnetic susceptibility of a system characterizes the response of this system to a magnetic field and gives a measure of the energy required to produce a net spin alignment in the direction of the field. It is defined as

$$
\chi=\left(\frac{\partial \mathcal{M}}{\partial \mathcal{H}}\right)_{\mathcal{H}=0}
$$

where $\mathcal{M}$ is the magnetization of the system per unit volume given by

$$
\mathcal{M}=\mu\left(\rho^{\uparrow}-\rho^{\downarrow}\right)=\mu \rho \Delta
$$

with $\mu$ the magnetic moment of a neutron, and $\mathcal{H}$ is the magnetic field which can be obtained from

$$
\mathcal{H}=\rho\left(\frac{\partial(E / N)}{\partial \mathcal{M}}\right)_{\mathcal{M}=0}=\frac{1}{\mu}\left(\frac{\partial(E / N)}{\partial \Delta}\right)_{\Delta=0} .
$$

Using Eqs. (7) and (8), the magnetic susceptibility can be written as

$$
\chi=\frac{\mu^{2} \rho}{\left(\frac{\partial^{2}(E / N)}{\partial \Delta^{2}}\right)_{\Delta=0}}
$$

where the second derivative can be taken at $\Delta=0$ if the field $\mathcal{H}$ is assumed to be small.

It is customary to study the magnetic susceptibility in terms of the ratio $\chi / \chi_{F}$, where $\chi_{F}$ is the magnetic susceptibility of a free Fermi gas, usually known as Pauli susceptibility. It can be straightforwardly obtained from Eq. (9) and the total energy per particle of the free Fermi gas

$$
\chi_{F}=\frac{\mu^{2} m}{\hbar^{2} \pi^{2}} k_{F}
$$

where the Fermi momentum $k_{F}=\left(3 \pi^{2} \rho\right)^{1 / 3}$ is related to $k_{F}^{\uparrow}$ and $k_{F}^{\downarrow}$ through the relations

$$
\begin{aligned}
& k_{F}^{\uparrow}=k_{F}(1+\Delta)^{1 / 3} \\
& k_{F}^{\downarrow}=k_{F}(1-\Delta)^{1 / 3} .
\end{aligned}
$$



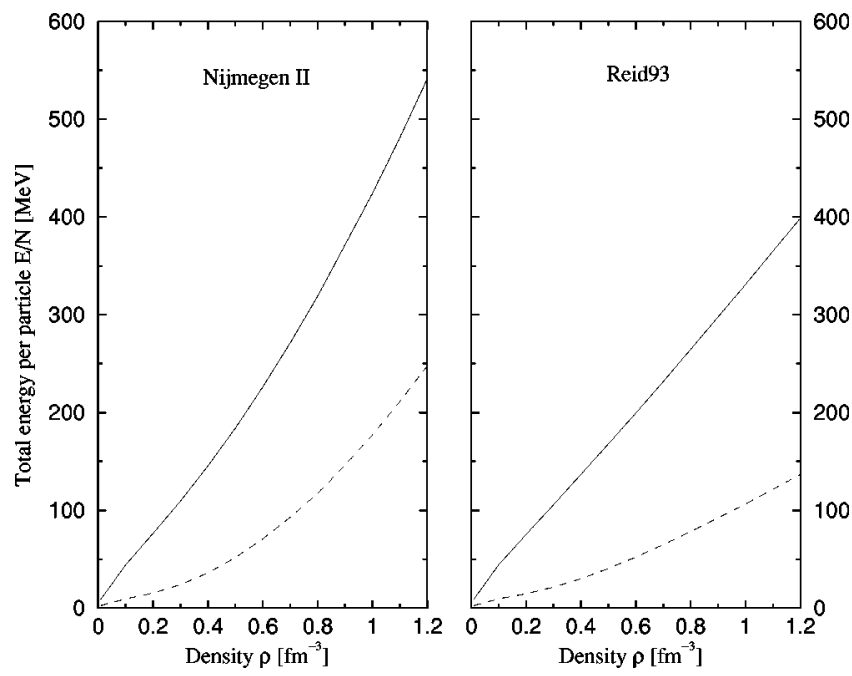

FIG. 1. Total energy per particle as a function of the density for totally polarized (solid lines) and nonpolarized (dashed lines) neutron matter. The left panel shows results for the Nijmegen II nucleon-nucleon interaction, whereas results on the right panel correspond to the Reid93 interaction.

\section{RESULTS}

The total energy per particle for totally polarized (solid lines) and nonpolarized (dashed lines) neutron matter is shown in Fig. 1 as a function of the density. Results for the Nijmegen II interaction are plotted on the left panel, whereas those corresponding to the Reid93 interaction are shown on the right panel. As can be seen from the figure, for both interaction models, totally polarized neutron matter is always more repulsive than nonpolarized neutron matter in all the density range explored. This additional repulsion can be understood, firstly, in terms of the kinetic energy contribution, which is larger in the totally polarized case than in the nonpolarized one. Secondly, in terms of the potential energy contribution because, due to symmetry arguments, all partial waves with even orbital angular momentum $L$ (some of them attractive, as the important ${ }^{1} S_{0}$ ) are excluded in totally polarized neutron matter. In order to illustrate this, we have plotted in Fig. 2 the separate kinetic (left panel) and potential (right panel) energy contributions for the Nijmegen II interaction model (similar results are obtained for the Reid93 one, but they are not included in order to make the discussion more clear). An interesting conclusion which can be inferred from these results is that a phase transition to a ferromagnetic state is not to be expected from our calculation. If such a transition would exist, a crossing of the energies of the totally polarized and the nonpolarized cases would be observed at some density, indicating that the ground state of the system would be ferromagnetic from that density on. As can be seen in Fig. 1, there is no sign of such a crossing and, on the contrary, it becomes less favorable as the density increases.

We have shown results for totally polarized and nonpolarized neutron matter. Let us consider now an intermediate situation in which not all the spins, but a part of them, are aligned in a given direction, and let us examine the dependence of the total energy per particle in the spin polarization
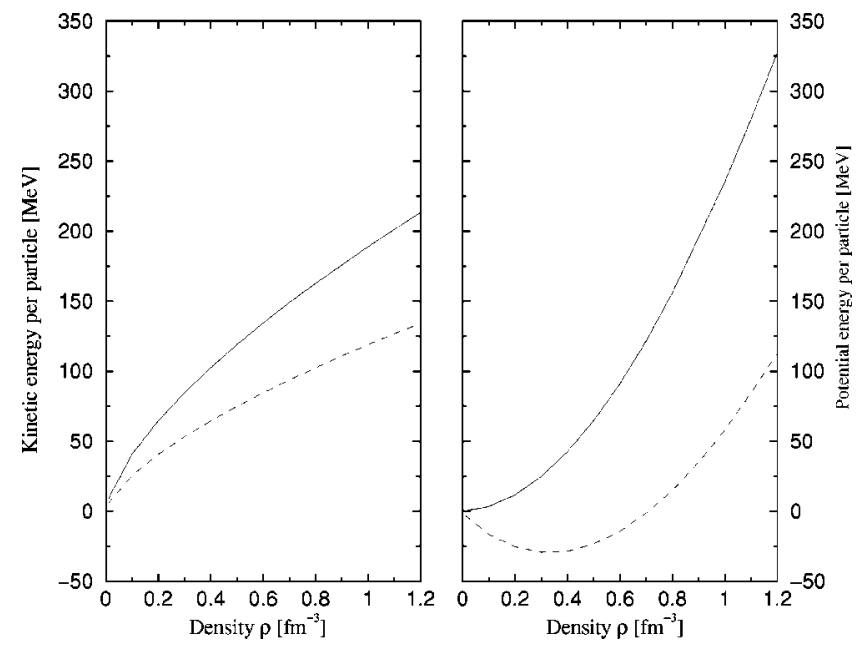

FIG. 2. Kinetic (left panel) and potential (right panel) energy contributions to the total energy per particle as a function of the density for totally polarized (solid lines) and nonpolarized (dashed lines) neutron matter. Results are shown for the Nijmegen II interaction.

$\Delta$. This dependence is shown in Fig. 3 for five different densities $\left(\rho_{0} / 2, \rho_{0}, 2 \rho_{0}, 5 \rho_{0}\right.$, and $7 \rho_{0}$, being $\rho_{0}=0.16 \mathrm{fm}^{-3}$ the saturation density of nuclear matter). As in Fig. 1, results for the Nijmegen (Reid93) are shown on the left (right) panel. Circles, squares, diamonds, and triangles correspond to our BHF results, whereas solid lines correspond to the parabolic approximation discussed below. As can be seen from this figure, and as it was expected, $E / N$ is symmetric in $\Delta$. It can also be seen in this figure that $E / N$ shows a minimum at $\Delta=0$ for all the densities considered, being this again an indication that the ground state of neutron matter is
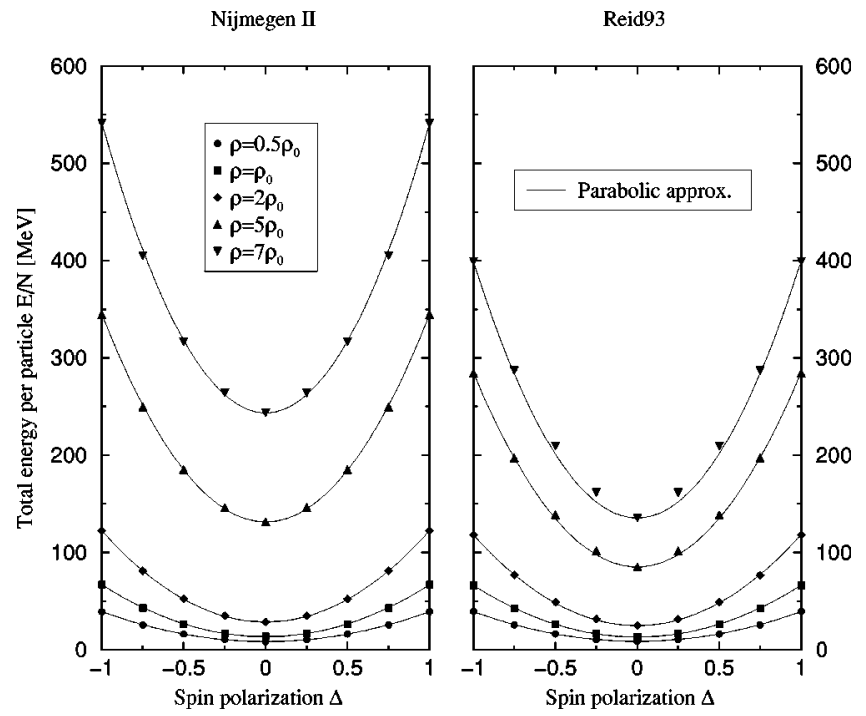

FIG. 3. Total energy per particle as a function of the spin polarization $\Delta$ for different densities. Results for the Nijmegen II (Reid93) interaction are shown in the left (right) panel. Circles, squares, diamonds, and triangles show our BHF results, whereas solid lines correspond to the parabolic approximation defined in Eq. (12). 
paramagnetic. Another interesting thing is to note that this dependence is up to a very good approximation parabolic, being this parabolic character only slightly lost at very large densities. Note that the kinetic energy contribution in fact follows a $(1+\Delta)^{5 / 3}+(1-\Delta)^{5 / 3}$ law, producing deviations from a parabolic behavior of at most $2 \%$. In the case of local interactions the dependence on the spin polarization $\Delta$ comes from the exchange terms and, if the interaction is a contact one, it is easy to see that this dependence is strictly parabolic. Finite range forces produce deviations from this law and the fact that these deviations are small for realistic nucleonnucleon interactions has useful consequences for the calculation of the magnetic susceptibility. Indeed, in the same spirit as it is done in nuclear matter to determine the symmetry energy, one can try to characterize the dependence of the energy per particle on the spin polarization in the following simple analytic form:

$$
\frac{E}{N}(\rho, \Delta)=\frac{E}{N}(\rho, 0)+a(\rho) \Delta^{2},
$$

where, assuming the quadratic dependence to be valid up to $|\Delta|=1$ as our results indicate, the value of $a(\rho)$ can be easily obtained for each density as the difference between the total energy per particle of totally polarized and nonpolarized neutron matter

$$
a(\rho)=\frac{E}{N}(\rho, \pm 1)-\frac{E}{N}(\rho, 0) .
$$

The magnetic susceptibility can be evaluated in a very simple way if the parabolic dependence of Eq. (12) is assumed, giving

$$
\chi(\rho)=\frac{\mu^{2} \rho}{2 a(\rho)} .
$$

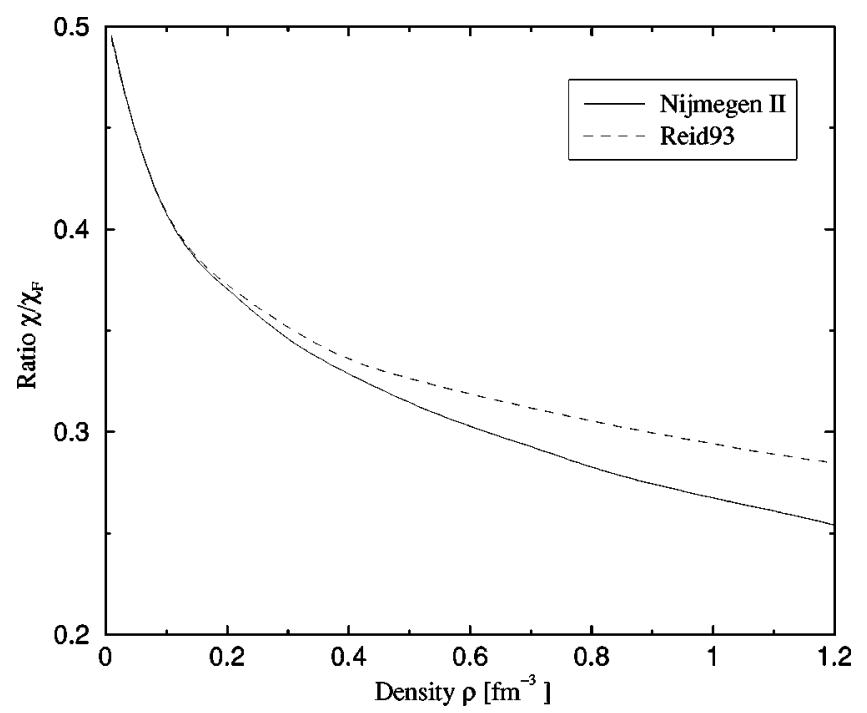

FIG. 4. Ratio $\chi / \chi_{F}$ as a function of the density. The solid line shows the result for the Nijmegen II interaction, while the dashed line corresponds to the one obtained with Reid93.
TABLE I. Magnetic susceptibility ratio $\chi / \chi_{F}$. Our BHF results, labeled Nijmegen II and Reid93, are compared with the AFDMC results of Fantoni et al. (Ref. [12]), labeled AU6' and AU8'. BHF results obtained with the standard discontinuous prescription are given in parentheses.

\begin{tabular}{ccccc}
\hline \hline$\rho / \rho_{0}$ & Nijmegen II & Reid93 & AU6' & AU8' \\
\hline 0.75 & $0.39(0.41)$ & $0.40(0.41)$ & 0.40 & \\
1.25 & $0.37(0.39)$ & $0.37(0.39)$ & 0.37 & 0.39 \\
2.0 & $0.34(0.37)$ & $0.35(0.38)$ & 0.33 & 0.35 \\
2.5 & $0.33(0.36)$ & $0.34(0.37)$ & 0.30 & \\
\hline \hline
\end{tabular}

In Fig. 4 the ratio $\chi / \chi_{F}$ is shown as a function of the density. The solid line shows the result for the Nijmegen II interaction, whereas the dashed line corresponds to the one obtained with Reid93. Starting from 1, the ratio decreases rapidly for small densities and more slowly as density increases. It can be inferred again from this figure that a ferromagnetic phase transition, which would be signaled by an infinite discontinuity giving rise to a change of sign in $\chi / \chi_{F}$, is not seen and not expected at larger densities either.

Finally, our results for $\chi / \chi_{F}$ are compared in Table I with those of the recent calculation performed by Fantoni et al. [12], shown in columns labeled AU6' and AU8'. As can be seen from the table, there is a very good agreement between these results and ours. For completeness, we show in parentheses the results obtained when the standard discontinuous prescription is used in solving the Bethe-Goldstone equation. In both prescriptions, the results are very similar, which is not surprising due to the fact that $\chi$ is obtained from an energy difference [see Eqs. (13) and (14)] which partly cancels the possible discrepancies. Only for densities larger than $2 \rho_{0}$, the discontinuous prescription results differ more than $10 \%$ from the continuous ones.

\section{SUMMARY AND CONCLUSIONS}

Employing realistic modern nucleon-nucleon interactions (Nijmegen II and Reid93) we have performed a BruecknerHartree-Fock calculation of spin polarized neutron matter properties. We have studied the total energy per particle of neutron matter as a function of the density and the spin polarization $\Delta$. We have found that in the range of densities explored (up to $7 \rho_{0}$ ) totally polarized matter is always more repulsive than nonpolarized matter, being this an indication that a phase transition of the system to a ferromagnetic state is not expected.

We have seen that the total energy per particle is not only symmetric on the spin polarization $\Delta$, as it was expected, but also parabolic in a very good approximation up to $|\Delta|=1$ even at high densities. This finding supports the calculation of the magnetic susceptibility of neutron matter by using only the energies of the spin symmetric and fully polarized systems.

Finally, we have calculated the magnetic susceptibility of the system as a function of the density, finding a very good agreement with a recent Monte Carlo calculation [12]. 


\section{ACKNOWLEDGMENTS}

This work was partially supported by the DGICYT Contract No. PB98-1247 and by the Generalitat de Catalunya Grant
No. 2000SGR00024 (Spain). The authors acknowledge useful discussions with Professor J. Navarro. One of the authors (I.V.) wishes to acknowledge support from the Istituto Nazionale di Fisica Nucleare (Italy).
[1] T. Gold, Nature (London) 218, 731 (1968).

[2] D. H. Brownell, Jr. and J. Callaway, Nuovo Cimento B 60, 169 (1969).

[3] M. J. Rice, Phys. Lett. 29A, 637 (1969).

[4] S. D. Silverstein, Phys. Rev. Lett. 23, 139 (1963).

[5] E. Østgaard, Nucl. Phys. A154, 202 (1970).

[6] J. W. Clark, Phys. Rev. Lett. 23, 1463 (1969).

[7] J. M. Pearson and G. Saunier, Phys. Rev. Lett. 24, 325 (1970).

[8] V. R. Pandharipande, V. K. Garde, and J. K. Srivastava, Phys. Lett. 38B, 485 (1972).

[9] S. O. Bäckmann and C. G. Källman, Phys. Lett. 43B, 263 (1973).

[10] A. D. Jackson, E. Krotscheck, D. E. Meltzer, and R. A. Smith, Nucl. Phys. A386, 125 (1982).
[11] A. Vidaurre, J. Navarro, and J. Bernabéu, Astron. Astrophys. 135, 361 (1984).

[12] S. Fantoni, A. Sarsa, and E. Schmidt, Phys. Rev. Lett. 87, 181 101 (2001).

[13] A. Smerzi, D. G. Ravenhall, and V. R. Pandharipande, Phys. Rev. C 56, 2549 (1997).

[14] S. C. Pieper, Microscopic Quantum Many-Body Theories and their Applications, Vol. 510 of Lecture Notes in Physics (Springer Verlag, Berlin, 1998), p. 337.

[15] V. G. J. Stoks, R. A. M. Klomp, C. P. F. Terheggen, and L. J. de Swart, Phys. Rev. C 49, 2950 (1994).

[16] H. Q. Song, M. Baldo, G. Giansiracusa, and U. Lombardo, Phys. Rev. Lett. 81, 1584 (1998). 